\title{
Recall of Physiology Knowledge Among Medical Interns: An Exploratory study in Riyadh, Saudi Arabia
}

\author{
Asmaa Mohammed AlMohanna, Muaawia A. Hamza*, Mohammed E. Suliman, Noran \\ Abdullah AlEssa, Said Y. Khatib and Abdallah Abdelwahid Saeed \\ Faculty of Medicine, King Fahad Medical City
}

The aim of the study was to explore the factors associated with the recall of basic medical physiology knowledge among medical interns and to determine the level of retained basic science knowledge.

Two hundred and forty interns, 114 females and 90 males, working in two major tertiary medical care centers, King Fahad Medical City (KFMC 29 students) and King Khalid University Hospital (KKUH), 117 students), in Riyadh city, participated in the study. An anonymous knowledge test with 10 validated multiple choice questions was developed specifically for this purpose. One hundred and forty-six interns (117 working at KKUH and 29 at KFMC) had graduated from medical schools adopting a conventional instructional system whereas 58 ( 3 from KKUH and 55 from KFMC had graduated from schools adopting an integrated system (Hybrid PBL). Fifty-two students (26\%) gained a score $\geq 60 \%$, whereas 152 students $(74 \%)$ obtained $<60 \%$ of the score. Higher scores were associated with younger age $(\square<0.01)$, traditional curriculum $(\square<0.001)$, interns from KKUH $(\square<0.001)$ candidates for postgraduate studies $((\square<0.02)$, There was no significant association between recall of physiology knowledge and all other variables studied, including gender. Multivariate analyses show that age and traditional curriculum are the only significant predictors of knowledge retention. Almost three-quarters of the interns scored less than $60 \%$ and higher scores were significantly associated with younger interns, traditional curriculum, working in $\mathrm{KKUH}$ and interns preparing for graduate studies. However, the difference between the two curricula disappears when the influence of hospital training is considered.

Key Words: Physiology, Knowledge retention, Curriculum, Interns, KSA

Acknowledgement: This study was funded by a generous grant form SRC, Faculty of Medicine, King Fahad Medical City, Riyadh, KSA

* Presenting author

This paper was published in the Adv Physiol Educ 42: 541-546, 2018 